\title{
Renal metastasis from cervical carcinoma presenting as a renal cyst: A case report
}

\author{
GANG FAN $^{1}$, YU XIE $^{1}$, XIAMING PEI $^{1}$, JIAN LEI $^{2}$, MINGJI YE ${ }^{1}$, GONGQIAN ZENG $^{1}$, \\ FEIPING $\mathrm{LI}^{3}$, YINGYING XIONG ${ }^{2}$ and WEIQIN HAN ${ }^{1}$ \\ Departments of ${ }^{1}$ Urology, ${ }^{2}$ Pathology and ${ }^{3}$ Radiology, Hunan Provincial Tumor Hospital, \\ The Affiliated Tumor Hospital of Xiangya Medical College, Central South University, \\ Changsha, Hunan 410013, P.R. China
}

Received December 21, 2014; Accepted August 5, 2015

DOI: $10.3892 / \mathrm{ol} .2015 .3690$

\begin{abstract}
In the present study, the case of a 51-year-old female with a metastatic tumor in the left kidney originating from cervical carcinoma, is reported. The patient had undergone chemoradiotherapy for stage IIB squamous-cell carcinoma of the uterine cervix 3 years earlier. Computed tomography (CT) identified low-density left renal nodules, which were diagnosed post-operatively as renal cysts during the follow-up conducted 2 years later. The next year, the patient was admitted to the Hunan Provincial Tumor Hospital (The Affiliated Tumor Hospital of Xiangya Medical College, Central South University, Changsha, Hunan, China) with a fever of unknown origin, left-sided flank pain and hematuria. CT examination detected irregular low-density nodules in the left kidney and heterogeneous enhancement on enhanced CT. Subsequently, the patient was subjected to a nephrectomy. Post-surgical analysis of subsequent biopsies indicated kidney tumor metastasis originating from cervical carcinoma. Renal metastases are rare in patients with cervical carcinoma. The present study reported a case of renal metastasis originating from cervical carcinoma and also reviewed previous case reports on patients presenting with this unusual type of cancer.
\end{abstract}

\section{Introduction}

Globally, 500,000 cases of cervical cancer are diagnosed per year, which accounts for $5 \%$ of all cases of cancer diagnosed worldwide. The majority of these cases $(>80 \%)$ occur in developing countries $(1,2)$. Direct invasion is the main method for the diffusion of cervical cancer, followed by lymphatic metastasis, whereas hematogenous diffusion rarely occurs (3). Tumor cell

Correspondence to: Professor Yu Xie, Department of Urology, Hunan Provincial Tumor Hospital, The Affiliated Tumor Hospital of Xiangya Medical College, Central South University, 283 Tongzipo Road, Changsha, Hunan 410013, P.R. China

E-mail:1715840770@qq.com

Key words: metastasis, cervical cancer, renal cyst, nephrectomy hematogenous diffusion is a typical symptom of terminal cervical cancer. Common sites of such diffusion are the lungs, bones, aorta, and the celiac and supraclavicular lymph nodes, whereas kidney metastases are rare (4). To the best of our knowledge, 9 cases of renal metastasis originating from cervical carcinoma have been reported thus far (5-13), and 5 of these were initially misdiagnosed as other kidney-associated diseases $(6,8,10-12)$. Currently, no general treatment consensus exists for the treatment of renal metastases originating from cervical carcinoma. In the present study, the case of a 51-year-old female with a metastatic renal tumor originating from cervical carcinoma is described, and the existing strategies for the treatment of this condition are discussed. Written informed consent was obtained from the patient's husband for publication of the present study.

\section{Case report}

In February 2011, a 48-year-old female who was diagnosed with stage IIB cervical squamous-cell carcinoma underwent chemoradiotherapy (pelvic radiation, 24 fractions at $200 \mathrm{cGy} /$ fraction; intraoperative radiotherapy, 7 fractions at $600 \mathrm{cGy} /$ fraction; and chemotherapy, $60 \mathrm{mg}$ docetaxel twice/week) in the Department of Gynecology, Hunan Provincial Tumor Hospital (The Affiliated Tumor Hospital of Xiangya Medical College, Central South University, Changsha, Hunan, China).

On June 19th, 2013, during follow-up abdominal computed tomography (CT) examination, a low-density cystic mass with homogenous density and clear borders was identified post-operatively in the left kidney, which was interpreted as a renal cyst (Fig. 1).

On July 22nd, 2014, the patient consulted the Hunan Provincial Tumor Hospital due to gross hematuria, a fever of unknown origin (FUO) and left abdominal pain. The findings at the time of admission were as follows: Axillary temperature, $38.8^{\circ} \mathrm{C}$ (normal, $36.0-37.0^{\circ} \mathrm{C}$ ); heart rate, 84 beats $/ \mathrm{min}$ (normal, 60-100 beats/min); and blood pressure, $103 / 73 \mathrm{mmHg}$ (normal, 120/90-90/60 mmHg). Laboratory studies revealed the following results: A decreased red blood cell count of $2.93 \times 10^{12}$ cells/1 (normal, 3.5-4.5×10 12 cells/1), a decreased level of hemoglobin level of $97 \mathrm{~g} / \mathrm{l}$ (normal, 110-150 g/l), tumor-specific growth factor (normal, $0-64 \mu \mathrm{mol} / \mathrm{l}$ ), carcinoembryonic antigen (normal, $\leq 5.00 \mathrm{ng} / \mathrm{ml}$ ), $\alpha$-fetoprotein (normal, 0-20 ng/l) and 


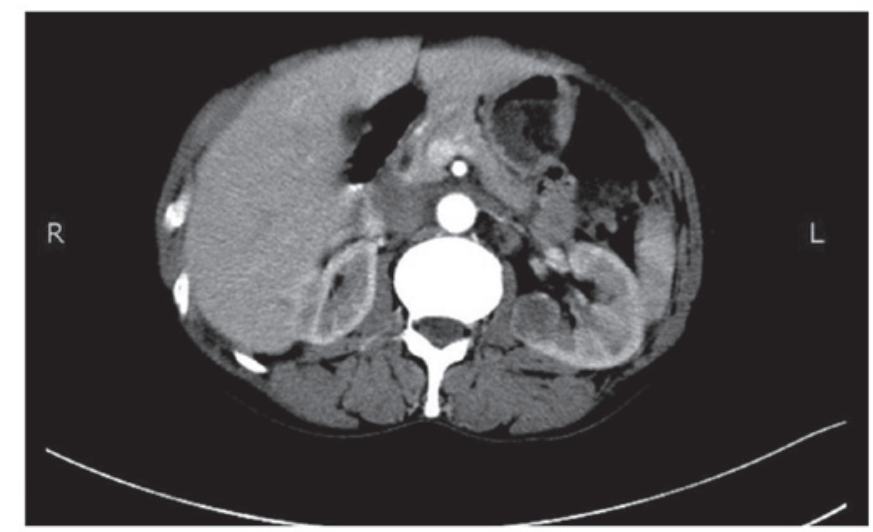

Figure 1. Enhanced axial computed tomography scan performed on June 19th, 2013, revealing a low-density cystic mass with homogenous density and clear borders in the right kidney.

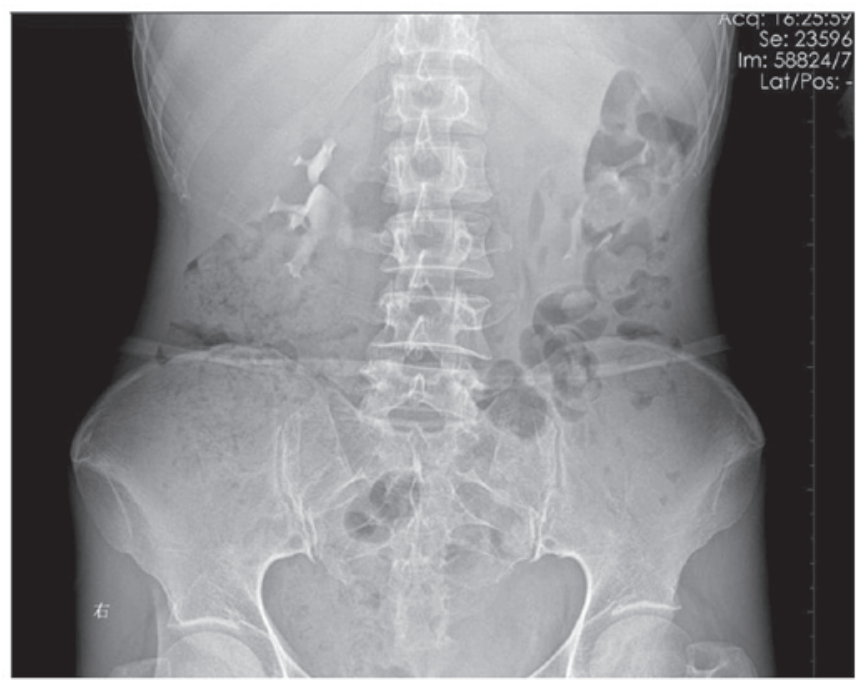

Figure 2. Intravenous pyelogram conducted on July 22nd, 2014, demonstrating distortion and compression of the upper pole of the left kidney.

human chorionic gonadotropin (normal, $<3.00 \mathrm{mIU} / \mathrm{l}$ ) levels of $60.60 \mu \mathrm{mol} / 1,0.92 \mathrm{mg} / 1,1.36 \mathrm{ng} / \mathrm{ml}$ and $0.35 \mathrm{mIU} / 1$, respectively, and high levels of high-sensitivity $\mathrm{C}$-reactive protein at $41.77 \mathrm{mg} / \mathrm{l}$ (normal, $<6.00 \mathrm{mg} / \mathrm{l}$ ).

Intravenous pyelogram demonstrated distortion and compression of the upper pole of the left kidney (Fig. 2). Abdominal ultrasound identified a $90 \times 60-\mathrm{mm}$ space-occupying lesion in the upper pole of the left kidney. Signals of intratumoral blood flow were detected by color Doppler ultrasound (Fig. 3). Abdominal CT detected irregular low-density nodules in the left kidney and heterogeneous enhancement on enhanced CT (Fig. 4). Additionally, the results of X-ray analysis were negative, the Eastern Cooperative Oncology Group performance status was 1 point (14) and the right renal function was adequate.

A left renal nephrectomy was performed 9 days after the admission date. The size of the phyma was $80 \times 60 \times 80 \mathrm{~mm}$. Pathological analysis showed that the sections of kidney were predominately composed of squamous cells that were similar to the pattern of the original cervical carcinoma (Fig. 5A). Immunohistochemical analysis indicated that the squamous
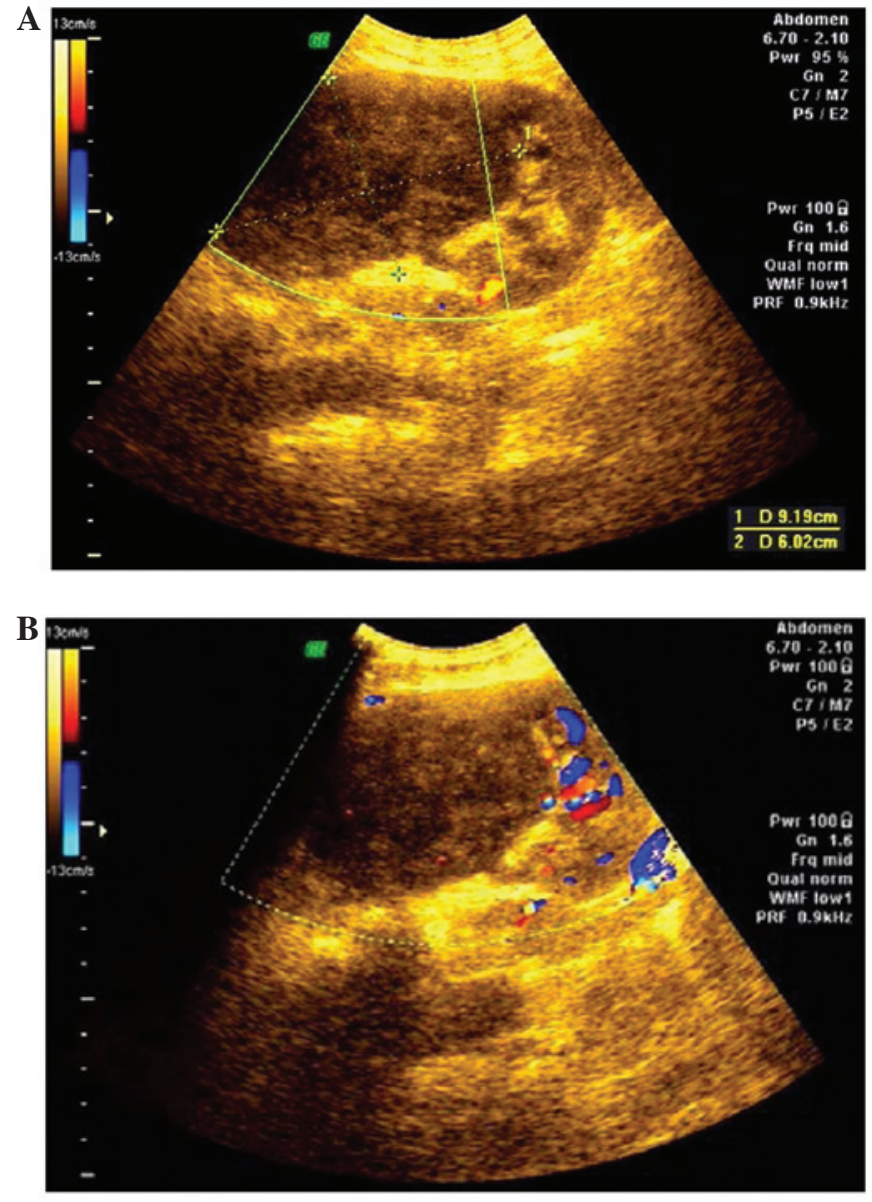

Figure 3. Color Doppler ultrasound of the left kidney performed on July 22,2014 , identifying (A) a 90x60-mm space-occupying lesion in the upper pole of the left kidney, and (B) blood flow signals in the tumor.

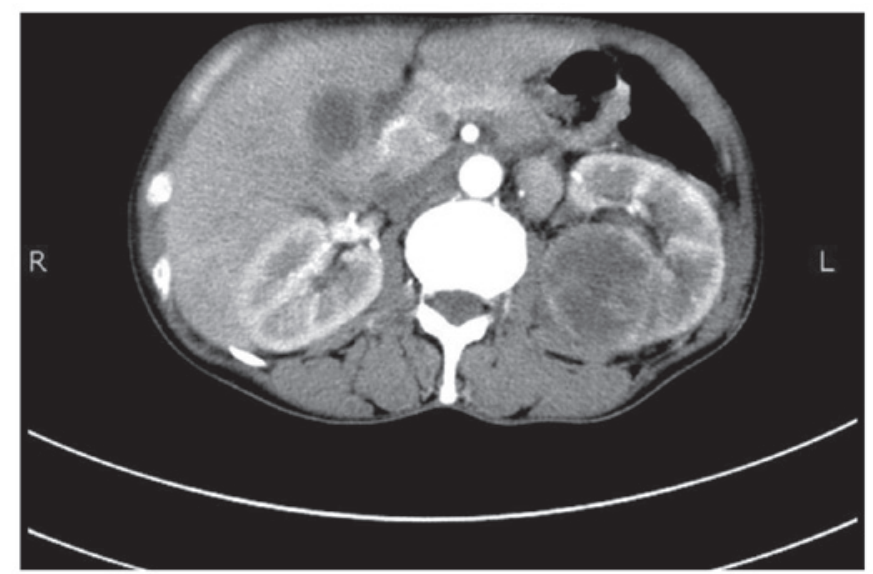

Figure 4. Abdominal computed tomography scan conducted on July 22nd, 2014, showing irregular low-density nodules with heterogeneous enhancement in the left kidney.

cell components were positive for cytokeratin 5/6 (Fig. 5B) and p63 (Fig. 5C). Thus, pathological and immunohistochemical examination confirmed a diagnosis of metastatic squamous cell carcinoma, which was histologically consistent with the original cervical carcinoma experienced by the patient 3 years prior.

On November 21st, 2014, the imaging results were positive for the disease in the lungs and multiple retroperitoneal lymph 
A

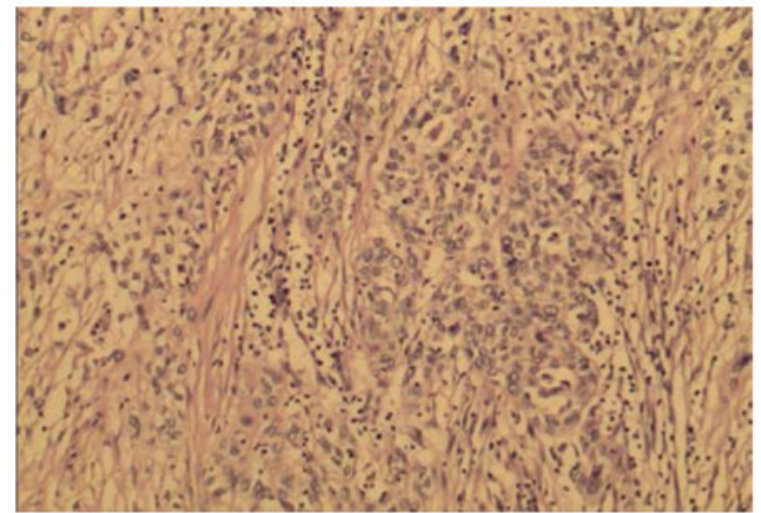

B

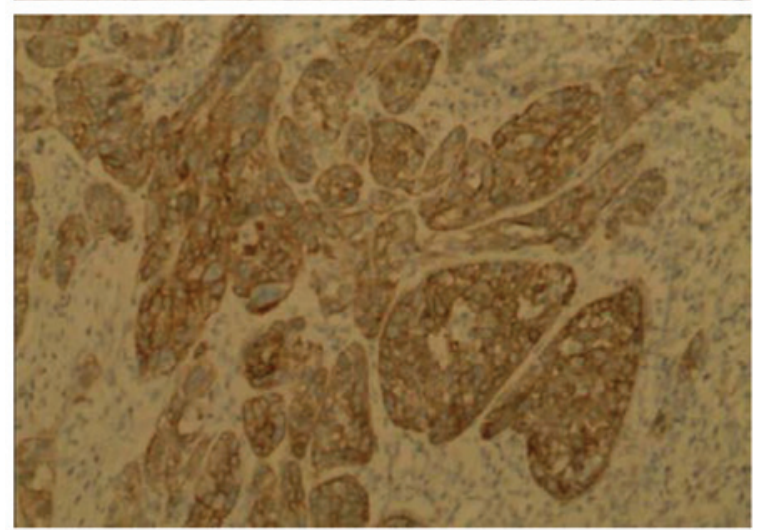

C

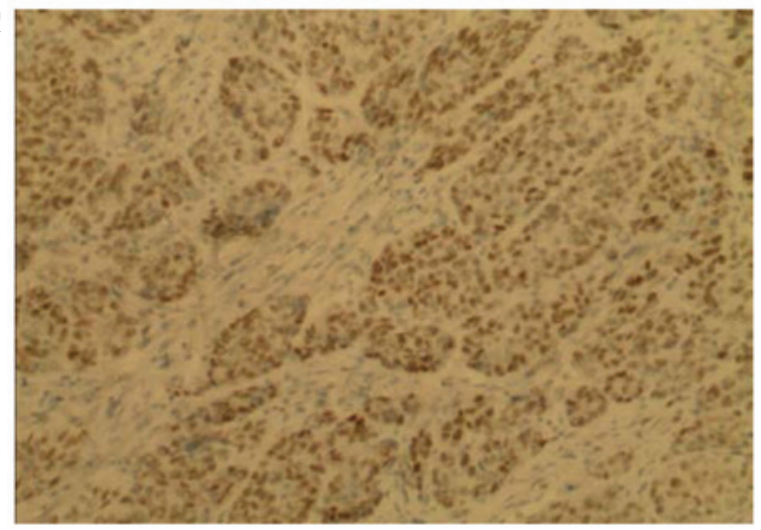

Figure 5. (A) Pathological examination revealing a squamous cell carcinoma that was similar to the pattern of the original cervical carcinoma (hematoxylin and eosin staining; magnification, x100). Also, immunohistochemical analysis demonstrating positive expression of (B) cytokeratin 5/6 and (C) p63 in the renal tumor (magnification, $\mathrm{x} 100$ ).

nodes. The patient consequently refused to continue with the treatment and was discharged from the hospital.

\section{Discussion}

Metastatic renal tumors are rare, and are mainly detected at autopsy. Klinger (15) reviewed 5,000 autopsies and identified 118 cases of kidney tumor metastases, of which, 2 cases (1.69\%) were secondary to cervical cancer. Similar findings were reported in the study by Wagle et al (16), which identified a rate of $2.5 \%$ for kidney metastases originating from cervical carcinoma from 4,413 autopsies.

Kidney tumor metastases are rare, and only 9 cases have been reported to originate from cervical carcinoma to date; the most recent case dating from 2013 (13). Differences exist among primary renal cell carcinomas and kidney metastases. Metastatic renal tumors generally present with diameters of $<4 \mathrm{~cm}$, and the majority are bilateral multiple lumps. Of the 9 cases reported thus far, 4 have been bilateral kidney tumors, and 5 unilateral. The majority of these cases (13) occurred within a 15-month period after diagnosis and primary treatment, with the longest period lasting 118 months (12).

In the present case, the patient experienced recurrence within 42 months of the initial diagnosis. Initially, a left renal cyst was suspected, based on the results of the CT examinations conducted 1 year after the primary treatment. However, the patient returned to the Hunan Provincial Tumor Hospital 1 year later, presenting with gross hematuria, FUO and left abdominal pain. Based on the clinical symptoms and diagnostic imaging at the time of admission, a metastatic renal tumor was suspected. The patient was then subjected to nephrectomy, and the subsequent pathological and immunohistochemical examinations confirmed squamous cell carcinoma.

Diagnoses of metastatic renal tumors mostly rely on the findings from radiographic examinations and the clinical history of the patient (17). Metastatic renal masses are not always easily characterized by CT, therefore potentially leading to misdiagnosis. Out of the 9 cases of metastatic renal tumors originating from cervical carcinoma that have been reported thus far (5-13), 5 were initially misdiagnosed as renal abscesses due to the symptoms displayed by the patients $(6,8,10-12)$, including fever $\left(37.5-41.0^{\circ} \mathrm{C}\right)$, abdominal pain and alterations in the levels of certain biochemical indicators. Lin et al (12) highlighted the difficulty in diagnosing renal metastases by radiographic examination, stating that the patients often experience FUO, pain and biochemical abnormalities, which may easily lead to misdiagnosis. Therefore, if a patient with FUO presents with a history of a primary neoplasm, a secondary renal tumor should be considered in the differential diagnosis of the renal mass lesion. For those patients in good condition, a renal biopsy is required in order to confirm the diagnosis of renal metastasis. Compared with the 9 previous cases reported in the literature, the patient in the present study exhibited a single lesion with a large volume $(80 \times 60 \times 80 \mathrm{~mm})$. The abdominal CT examination conducted on June 2013 identified a low-density nodule in the left kidney. However, the clinical symptoms were insignificant at the time, and the nodule was misdiagnosed as a renal cyst. Notably, the patient experienced recurrence 13 months later, and exhibited significant clinical symptoms, including pain, fever and hematuria.

In patients with advanced cervical cancer, chemoradiotherapy and surgery plus chemotherapy are the main treatment options (18). However, patients with metastases derived from cervical carcinoma present with a poor prognosis despite chemoradiation and surgical treatment. The overall survival was $<12$ months for the previously reported 9 patients who underwent chemoradiotherapy or surgery for the treatment of renal metastases derived from cervical carcinoma. Ishihara et al (19) proposed that a kidney resection was more effective than antitumor therapy for the treatment of renal metastases originating from cervical carcinoma, since a kidney resection may reduce the symptoms of pain and improve the quality of life in these patients. Ogose et al (20) proposed a similar approach for the treatment of kidney metastases derived from osteosarcoma. However, surgery was unable to improve the survival rates of 
these patients. In the present study, the symptoms of the patient, including hematuria, fever and abdominal pain, were significantly relieved following nephrectomy. Furthermore, positive images of multiple organs without serious complications were observed 4 months after nephrectomy.

In conclusion, nephrectomy should be the preferred method for the treatment of kidney metastases derived from cervical carcinoma if the metastatic lesion is located unilaterally in the kidney. However, this treatment may only relieve the clinical symptoms of the patient. Conservative treatments, such as chemoradiation, may be an option in cases of bilateral metastatic renal tumors or in those presenting with other widespread lesions. In the present case study, the patient presented with cervical carcinoma and metastasis to the left kidney, which was initially misdiagnosed as a renal cyst. However, the cyst was subsequently confirmed to be a renal metastasis originating from cervical carcinoma.

\section{Acknowledgements}

The present study was supported by the Fundamental Research Funds for the Central Universities of Central South University (grant no. 2177/72150050625).

\section{References}

1. Jin XW, Sikon A, Yen-Lieberman B: Cervical cancer screening: Less testing, smarter testing. Cleve Clin J Med 78: 737-747, 2011.

2. Jemal A, Bray F, Center MM, Ferlay J, Ward E and Forman D: Global cancer statistics. CA Cancer J Clin 61: 69-90, 2011.

3. Benedetti-Panici P, Maneschi F, D'Andrea G, Cutillo G, Rabitti C, Congiu M, Coronetta F and Capelli A: Early cervical carcinoma. Cancer 88: 2267-2274, 2000.

4. Massad LS, Einstein MH, Huh WK, Katki HA, Kinney WK, Schiffman M, Solomon D, Wentzensen N and Lawson HW; 2012 ASCCP Consensus Guidelines Conference: 2012 updated consensus guide-lines for the management of abnormal cervical cancer screening tests and cancer precursors. Obstet Gynecol 121 829-846, 2013

5. Roy JB and Walton KN: Secondary tumors of the kidney. J Urol 103: 411-413, 1970.

6. Reznicek SB and Fallon B: Metastatic cervical carcinoma masquerading as bilateral renal abscesses. Urology 25: 174-175, 1985 .
7. Nagaoka S, Yamasaki A, Fuziwara E, Hayashi M, Nakano H and Nihira H: A case of bilateral metastatic renal tumor originating in a cervical carcinoma. Urol Int 41: 219-221, 1986.

8. Vilain C, Chaubard T, Gauthier JR, Adoue D and Le Tallec Y: Febrile secondary cancer of the kidney. Uncommon manifestation of isolated metastasis of epithelioma of the uterine cervix without pelvic recurrence. Ann Med Interne (Paris) 139: 440-442, 1988 (In French).

9. Koike H, Okamoto T, Tanji S, Fujioka T, Kubo T and Ohhori T: Two cases of metastatic renal tumor. Acta Urologica Japonica 35, 475-479, 1989.

10. de La Taille A, Bertrand P, Lemaitre L, Rigot JM and Mazeman E: Bilateral secondary renal neoplasm mimicking renal abscesses. Eur Urol 31, 249-250, 1997.

11. Takahashi A, Adachi H, Iwasawa A, Hirose T, Tsukamoto T, Hata $\mathrm{E}$ and Nomura Y: Metastatic cervical carcinoma mimicking kidney abscess. Int J Urol 5: 377-378, 1998.

12. Lin CM, Sun GH, Lee SS, Yu DS, Chang SY and Wu ST: Remote metastatic cervical carcinoma to kidneys mimicking bilateral renal abscesses. Eur J Cancer Care (Engl) 16: 526-528, 2007.

13. Jeon SW, Kim SH and Kwon SY: Renal metastasis from primary cervical cancer: A case report. J Korean Soc Radiol 68: 483-487, 2013.

14. Oken MM, Creech RH, Tormey DC, Horton J, Davis TE, McFadden ET and Carbone PP: Toxicity and response criteria of the Eastern Cooperative Oncology Group. Am J Clin Oncol 5: 649-655, 1982.

15. Klinger ME: Secondary tumors of the genito-urinary tract. J Urol 65: 144-153, 1951.

16. Wagle DG, Moore RH and Murphy GP: Secondary carcinomas of the kidney. J Urol 114: 30-32, 1975.

17. Ferrozzi F, Bova D, De Chiara F, Garlaschi G and Bassi P: CT of renal metastatic disease. A pictorial essay. Clin Imaging 19: 60-64, 1995.

18. Benedetti-Panici P, Greggi S, Colombo A, Amoroso M, Smaniotto D, Giannarelli D, Amunni G, Raspagliesi F, Zola P Mangioni C and Landoni F: Neoadjuvant chemotherapy and radical surgery versus exclusive radiotherapy in locally advanced squamous cell cervical cancer: Results from the Italian multicenter randomized study. J Clin Oncol 20: 179-188, 2002.

19. Ishihara S, Kobayashi S, Yamaha M, Takeuchi T, Kuriyama M, Ban Y, Kawada Y, Takahashi Y, Horie M and Isogai K: Metastatic renal tumor from the lung with regional lymph node involvement: A case report. Hinyokika Kiyo 36: 51-54, 1990.

20. Ogose A, Morita T, Emura I, Nemoto K and Hirata Y: Osteosarcoma metastatic to the kidneys without lung involvement. Jpn J Clin Oncol 29: 395-398, 1999. 